\title{
The Derivation of Structural Properties of LM-g Splines by Necessary Condition of Optimal Control
}

\author{
Xiongwei Liu, Xinjian Zhang, and Lizhi Cheng \\ College of Science, National University of Defense Technology, Changsha, Hunan 410073, China \\ Correspondence should be addressed to Xinjian Zhang; csxjzhang@163.com
}

Received 24 November 2013; Accepted 24 April 2014; Published 30 April 2014

Academic Editor: Ahmet Yasar Ozban

Copyright (C) 2014 Xiongwei Liu et al. This is an open access article distributed under the Creative Commons Attribution License, which permits unrestricted use, distribution, and reproduction in any medium, provided the original work is properly cited.

\begin{abstract}
The structural properties of LM-g splines are investigated by optimization and optimal control theory. The continuity and structure of LM-g splines are derived by using a class of necessary conditions with state constraints of optimal control and the relationship between LM-g interpolating splines and the corresponding L-g interpolating splines. This work provides a new method for further exploration of LM-g interpolating splines and its applications in the optimal control.
\end{abstract}

\section{Introduction}

Spline smoothing is widely used in many application areas as a basic tool of applied mathematics and a class of powerful and flexible modeling techniques. The operator spline function theory provides new descriptions and numerical methods for some optimal control problems [1-3]. Splines defined in terms of one linear differential operator are called L-g splines. The structural and continuity properties of these splines were discussed in detail in $[4,5]$. They play an important role in the approximation of functions and optimal control. Splines defined in terms of two differential operators were first considered by de Figueiredo [6] and were called LM-g splines. He defined LM-g splines by separating Green's function and showed their applications in the estimation of stochastic processes, minimum-energy controls, digital signal processing, and system modeling. However, it was difficult to discuss the structural and continuity properties of LM-g splines by the method of separating Green's function. Soon afterwards, Weinert et al. [7] redefined LM-g splines with certain integrodifferential operators in terms of the system inverses. In [7], these splines were indicated as sample functions of a certain type of autoregressive-moving average (ARMA) stochastic processes, so they were also called ARMA splines. This stochastic correspondence was used to develop the recursive algorithms for ARMA splines, interpolating splines, and smoothing splines [5, 8]. Zhang and Fang [9] established a new recursive algorithm for LM-g splines by using system inverses and reproducing kernels, and based on this, a recursive algorithm for the minimum-energy control of a class of linear systems was also obtained.

Although LM-g splines have been applied extensively in many fields and some recursive algorithms for which these splines have been developed, a systematic research on the structural and continuity properties of these splines has not been done. It is a quite difficult task to discuss the structural and continuity properties of abstract operator splines. Opfer and Oberle [10] and Fredenhagen et al. [11] presented a derivation of cubic splines with obstacles by the optimal control criteria. Afterwards, this method was used to deduce the structural and continuity properties of generalized interpolating splines with obstacles [12]. Takahashi and Martin [13] considered and made the generalized splines by solving an optimal control problem for linear systems. Egerstedt and Martin [2] showed how splines arose naturally in the theory of linear control systems from the latest methods and applications and by using the tools of optimization over vector spaces, demonstrated how splines were a consequence of the optimization problem, and revealed many natural relations among control theory, numerical analysis, and statistics. Alhanaty and Bercovier [14] introduced optimal control methods over reparametrization for curve and surface design. Zhang and Liu $[15,16]$ considered the structural and continuity characteristics of the interpolating splines defined by 
differential operator by means of the optimal control criteria. As a generalization of the proposed methods, the structural and continuity properties of LM-g splines, or LM-g splines with obstacles, are deduced by optimal control theory in the present paper. The investigations in this paper further reveal the profound relations between splines and optimal control theory.

\section{System Inverse and LM-g Spline}

Let $L$ and $M$ be the linear differential operators defined by

$$
\begin{aligned}
& L=D^{n}+a_{n-1} D^{n-1}+a_{n-2} D^{n-2}+\cdots+a_{1} D+a_{0}, \\
& M=c_{m} D^{m}+c_{m-1} D^{m-1}+\cdots+c_{1} D+c_{0},
\end{aligned}
$$

where $a_{i}(i=0,1, \ldots, n-1), c_{j}(j=0,1, \ldots, m)$ are all constants, $c_{m} \neq 0, m<n$.

As shown in [9], $L f(t)=M u(t)$ can be written as

$$
\begin{aligned}
& \dot{x}(t)=A x(t)+\tilde{b} u(t), \\
& f(t)=c x(t),
\end{aligned}
$$

where

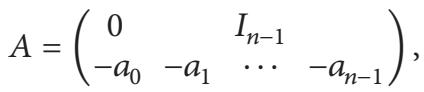

$$
\begin{aligned}
& x(t)=\left(x_{1}(t), x_{1}(t), \ldots, x_{n}(t)\right)^{T} \text {, } \\
& c=(1,0, \ldots, 0), \quad \widetilde{b}=\left(0, \ldots, 0, b_{\alpha}, b_{\alpha+1}, \ldots, b_{n}\right)^{T}, \\
& \alpha=n-m, \\
& b_{\alpha}=c_{m}, \quad b_{\alpha+j}=c_{m-j}-\sum_{k=0}^{j-1} a_{n-j+k} b_{\alpha+k}, \\
& j=1,2, \ldots, m \text {. }
\end{aligned}
$$

Let $W_{2}^{k}[0,1]$ be the linear space defined by

$$
\begin{aligned}
W_{2}^{k}[0,1]=\{ & f(t), t \in[0,1]: f^{(k-1)} \text { absolutely continuous } \\
& \text { and } \left.f^{(k)} \in L^{2}[0,1]\right\} .
\end{aligned}
$$

Suppose that $P$ is the linear operator mapping the output $f(t)$ to the input $u(t)$ of system (3); that is,

$$
P f(t)=u(t) \quad \text { or } \quad L f(t)=M u(t), \quad t \in[0,1] .
$$

From [17] and the inversion of system (3), we know that $P$ is a bounded linear integral-differential operator that maps $W_{2}^{\alpha}[0,1]$ onto the space $L^{2}[0,1]$ of square-integrable functions on the interval $[0,1]$. Suppose that $\left\{l_{j}\right\}_{j=1}^{N}(N \geq$ $n$ ) are linear functionals that are continuous and linearly independent of $W_{2}^{\alpha}[0,1]$. Let $\left\{r_{j}\right\}_{j=1}^{N}$ be real numbers and $U_{\alpha}(r)=\left\{f \in W_{2}^{\alpha}[0,1]: l_{j} f=r_{j}, 1 \leq j \leq N\right\}$. From [6, 7], we know if $\left\{l_{j}\right\}_{j=1}^{\alpha}$ are linearly independent of ker $P=\{f \in$ $\left.W_{2}^{\alpha}[0,1]: P f=0\right\}$, the solution of the minimization problem

$$
\int_{0}^{1}[P \sigma(t)]^{2} d t=\min _{f \in U_{\alpha}(r)} \int_{0}^{1}[P f(t)]^{2} d t, \quad \sigma \in U_{\alpha}(r)
$$

always exists and is unique. The solution $\sigma(t)$ is usually called LM-g interpolating spline.

For the convenience of discussing the structural and continuity properties of LM-g spline $\sigma(t)$ and its applications, we will restrict attention to a rather broad class of constraint functionals $\left\{l_{j}\right\}_{j=1}^{N}$, called extended Hermite-Birkhoff (EHB) functionals, which have the form

$$
\begin{aligned}
& l_{j} f=\sum_{k=1}^{\gamma_{j}} \alpha_{j k} f^{(k-1)}\left(t_{j}\right), \\
& j=1,2, \ldots, N, 1 \leq \gamma_{j} \leq \alpha,
\end{aligned}
$$

where $\alpha_{j k}$ are constants, $0=t_{1}<t_{2}<\cdots<t_{N}=1$.

From (1), we define

$$
\begin{array}{r}
L_{0}^{*}=I \text { (identity operator), } \quad L_{j}^{*}=-D L_{j-1}^{*}+a_{n-j}, \\
j=1,2, \ldots, n .
\end{array}
$$

The operators $\left\{L_{j}^{*}\right\}_{j=1}^{n}$ are called the partial adjoint operator of $L . L^{*}=L_{n}^{*}$ is called the adjoint operator of $L$.

\section{Structural Properties of LM-g Spline}

According to the optimal control theory of linear systems, the minimization problem (9) is to find a control function $u(t)$ which is piecewise smooth with certain conditions to minimize the objective function

$$
J(u)=\frac{1}{2} \int_{0}^{1} u^{2}(t) d t
$$

with the constraints

$$
\begin{gathered}
\dot{x}(t)=A x(t)+\tilde{b} u(t), \\
F_{j}\left[x\left(t_{j}\right)\right]=\sum_{k=1}^{\gamma_{j}} \alpha_{j k} x_{k}\left(t_{j}\right)-r_{j}=0, \\
1 \leq \gamma_{j} \leq \alpha, 1 \leq j \leq N .
\end{gathered}
$$

From the necessary conditions of the optimal control with state constraints $[11,12]$, we know that there exist piecewise continuously differentiable Lagrange multipliers $\lambda(t)=\left(\lambda_{1}(t), \lambda_{2}(t), \ldots, \lambda_{n}(t)\right)$ and constants $\theta_{1}, \theta_{2}, \ldots, \theta_{N}$, such that, for the Hamiltonian function

$$
\widetilde{H}=\frac{1}{2} u^{2}(t)+\lambda(t)(A x(t)+\widetilde{b} u(t))
$$

and the state constraints (14), the following properties hold.

Adjoint differential equation:

$$
\begin{array}{r}
\dot{\lambda}(t)=-\frac{\partial \widetilde{H}}{\partial x}=-\left(\frac{\partial \widetilde{H}}{\partial x_{1}}, \frac{\partial \widetilde{H}}{\partial x_{2}}, \ldots, \frac{\partial \widetilde{H}}{\partial x_{n}}\right), \\
t \neq t_{j}, j=1,2, \ldots, N .
\end{array}
$$




\section{Transversality conditions:}

$$
\begin{aligned}
\lambda(0) & =-\frac{\partial}{\partial x(0)}\left[\theta_{1} F_{1}(x(0))\right], \\
\lambda(1) & =\frac{\partial}{\partial x(1)}\left[\theta_{N} F_{N}(x(1))\right], \\
\lambda\left(t_{j}^{+}\right) & =\lambda\left(t_{j}^{-}\right)-\frac{\partial}{\partial x\left(t_{j}\right)}\left[\theta_{j} F_{j}\left(x\left(t_{j}\right)\right)\right], \\
& j=2,3, \ldots, N-1 .
\end{aligned}
$$

Minimum principle: let $\left(\widetilde{u}^{*}(t), x^{*}(t)\right)$ be the solution pair of the system (12)-(14) and let $\lambda^{*}(t)$ be the solution of (16) satisfying conditions (17); then

$$
\widetilde{u}^{*}(t)=\min _{u \in \mathbb{R}} \widetilde{H}\left(t, x^{*}(t), u, \lambda^{*}(t)\right)
$$

From (3)-(9) and (12)-(14), we have

$$
\sigma(t)=x_{1}^{*}(t), \quad L \sigma(t)=M \tilde{u}^{*}(t) \text { or } P \sigma(t)=\tilde{u}^{*}(t) .
$$

Theorem 1. Let $\sigma(t)$ be the LM-g interpolating spline defined by (9), $L \sigma(t)=M \tilde{u}^{*}(t)$, and let

$$
v(t)=-\lambda_{n}^{*}(t)
$$

then

(i) $L^{*} P \sigma(t)=0$, for $t \neq t_{j}(j=1,2, \ldots, N)$;

(ii) $\tilde{u}^{*(i)}(a)=0\left(0 \leq i \leq \alpha-\gamma_{1}-1\right), \tilde{u}^{*(i)}(b)=0(0 \leq$ $\left.i \leq \alpha-\gamma_{N}-1\right), \tilde{u}^{*(i)}\left(t_{j}^{+}\right)-\tilde{u}^{*(i)}\left(t_{j}^{-}\right)=0,0 \leq i \leq$ $\alpha-\gamma_{j}-1,2 \leq j \leq N-1$;

(iii) $\sigma^{(i)}(a)=0\left(0 \leq i \leq 2 \alpha-\gamma_{1}-1\right), \sigma^{(i)}(b)=0(0 \leq i \leq$ $\left.2 \alpha-\gamma_{N}-1\right), \sigma^{(i)}\left(t_{j}^{+}\right)-\sigma^{(i)}\left(t_{j}^{-}\right)=0,0 \leq i \leq 2 \alpha-\gamma_{j}-1$, $2 \leq j \leq N-1$;

(iv) $L_{n-i}^{*}\left[v\left(t_{j}^{+}\right)-v\left(t_{j}^{-}\right)\right]=\theta_{j} \alpha_{j i}, \quad 1 \leq i \leq \gamma_{j}, 2 \leq j \leq N-1$.

Proof. From (3)-(6) and (15), we can get

$$
\begin{aligned}
\widetilde{H}= & \frac{1}{2} u^{2}-a_{0} \lambda_{n} x_{1}+\sum_{k=2}^{n}\left(\lambda_{k-1}-a_{k-1} \lambda_{n}\right) x_{k} \\
& +\left(\sum_{k=\alpha}^{n} b_{k} \lambda_{k}\right) u .
\end{aligned}
$$

Moreover, the adjoint differential equation leads to

$$
\begin{array}{r}
\dot{\lambda}_{1}(t)=a_{0} \lambda_{n}(t), \quad \dot{\lambda}_{j}(t)=a_{j-1} \lambda_{n}(t)-\lambda_{j-1}(t), \\
j=2,3, \ldots, n .
\end{array}
$$

Thus, $-\dot{\lambda}_{n}(t)+a_{n-1} \lambda_{n}(t)=\lambda_{n-1}(t)$ for $j=n$. From (11), $L_{1}^{*} \lambda_{n}(t)=\lambda_{n-1}(t)$. Then, we have

$$
L_{2}^{*} \lambda_{n}=-D L_{1}^{*} \lambda_{n}+a_{n-2} \lambda_{n}=-\dot{\lambda}_{n-1}(t)+a_{n-2} \lambda_{n} .
$$

From (22), we have

$$
-\dot{\lambda}_{n-1}(t)+a_{n-2} \lambda_{n}(t)=\lambda_{n-2}(t) .
$$

Substituting (24) into (23), one gets $L_{2}^{*} \lambda_{n}(t)=\lambda_{n-2}(t)$. Similarly, we can obtain

$$
\begin{gathered}
L_{1}^{*} \lambda_{n}=\lambda_{n-1}, \quad L_{2}^{*} \lambda_{n}=\lambda_{n-2}, \ldots, L_{n-1}^{*} \lambda_{n}=\lambda_{1}, \\
L^{*} \lambda_{n}=0 .
\end{gathered}
$$

According to the minimum principle and the necessary conditions of extremum, we know that $\widetilde{u}^{*}(t)$ is a solution of $\partial \widetilde{H} / \partial u=0$; thus,

$$
\widetilde{u}^{*}(t)=-b_{\alpha} \lambda_{\alpha}^{*}(t)-b_{\alpha+1} \lambda_{\alpha+1}^{*}(t)-\cdots-b_{n} \lambda_{n}^{*}(t) .
$$

Substituting (25) into (26) and noting $\alpha=n-m$, we have

$$
\begin{aligned}
\tilde{u}^{*}(t)=( & b_{n-m} L_{m}^{*}+b_{n-m+1} L_{m-1}^{*} \\
& \left.+\cdots+b_{n-1} L_{1}^{*}+b_{n}\right)\left(-\lambda_{n}^{*}(t)\right)
\end{aligned}
$$

Since $a_{j}(j=0,1, \ldots, n-1)$ are constants, it follows from (11) that, for $j=1,2, \ldots, n$,

$$
\begin{aligned}
L_{j}^{*}= & (-1)^{j} D^{j}+(-1)^{j-1} a_{n-1} D^{j-1} \\
& +(-1)^{j-2} a_{n-2} D^{j-2}+\cdots-a_{n-j+1} D+a_{n-j} .
\end{aligned}
$$

Substituting these expressions into (27) and recalling (3), it can be deduced that

$$
\begin{aligned}
& \tilde{u}^{*}(t)=\left((-1)^{m} c_{m} D^{m}+(-1)^{m-1} c_{m-1} D^{m-1}\right. \\
&\left.+\cdots-c_{1} D+c_{0}\right)\left(-\lambda_{n}(t)\right) \\
&=- M^{*} \lambda_{n}(t)=M^{*} v(t)
\end{aligned}
$$

where $M^{*}$ is the adjoint of $M$. Then, $P \sigma(t)=M^{*} v(t)$. From (20) and (25), we have $L^{*} v(t)=0$. Since $M^{*}$ and $L^{*}$ are constant coefficient linear differential operators, they are exchangeable. Hence, (i) holds.

By the transversality conditions (17), we have

$$
\begin{aligned}
& \lambda(0)=-\theta_{1}\left(\alpha_{11}, \alpha_{12}, \ldots, \alpha_{1 \gamma_{1}}, 0, \ldots, 0\right), \\
& \lambda(1)=\theta_{N}\left(\alpha_{N 1}, \alpha_{N 2}, \ldots, \alpha_{N \gamma_{N}}, 0, \ldots, 0\right), \\
& \lambda\left(t_{j}^{+}\right)=\lambda\left(t_{j}^{-}\right)-\theta_{j}\left(\alpha_{j 1}, \alpha_{j 2}, \ldots, \alpha_{j \gamma_{j}}, 0, \ldots, 0\right), \\
& j=2,3, \ldots, N-1 .
\end{aligned}
$$

Combining (25), we can get

$$
\begin{array}{r}
L_{0}^{*} v(0)=0, \quad L_{1}^{*} v(0)=0, \ldots, L_{n-\gamma_{1}-1}^{*} v(0)=0, \\
L_{0}^{*} v(1)=0, \quad L_{1}^{*} v(1)=0, \ldots, L_{n-\gamma_{N}-1}^{*} v(1)=0, \\
L_{i}^{*}\left[v\left(t_{j}^{+}\right)-v\left(t_{j}^{-}\right)\right]=0, \quad i=0,1, \ldots, n-\gamma_{j}-1, \\
j=2,3, \ldots, N-1 .
\end{array}
$$


It is easy to know from (11) that

$$
\begin{aligned}
& v^{(i)}(0)=0 \quad\left(i=0,1, \ldots, n-\gamma_{1}-1\right), \\
& v^{(i)}(1)=0 \quad\left(i=0,1, \ldots, n-\gamma_{N}-1\right), \\
& v^{(i)}\left(t_{j}^{+}\right)-v^{(i)}\left(t_{j}^{-}\right)=0, \quad i=0,1, \ldots, n-\gamma_{j}-1, \\
& j=2,3, \ldots, N-1 .
\end{aligned}
$$

Now, (ii) can follow from (29).

From (20) and (25), $L_{n-i}^{*} v(t)=-\lambda_{i}^{*}(t)(1 \leq i \leq n-1)$. Then, (30) shows that (iv) is true. (iii) can follow from (ii) and (19).

Hence, we complete the proof of Theorem 1.

\section{Relationship between LM-g Spline and L-g Spline}

Let $\left\{l_{j}\right\}_{j=1}^{N}(N \geq n)$ be linearly independent functionals on the space $W_{2}^{n}[0,1]$ and $U_{n}(r)=\left\{f \in W_{2}^{n}[0,1]: l_{j} f=r_{j}, 1 \leq\right.$ $j \leq N\}$. If $s(t)$ is a solution of the minimization problem

$$
\int_{0}^{1}[L s(t)]^{2} d t=\min _{f \in U_{n}(r)} \int_{0}^{1}[L f(t)]^{2} d t, \quad s \in U_{n}(r),
$$

it is called L-g interpolating spline [4].

If $\left\{l_{j}\right\}_{j=1}^{N}(N \geq n)$ are EHB functionals defined by (10), then solving the minimization problem (33) is to find a control function $u(t)$ which satisfies certain conditions of piecewise smoothness to minimize the functional

$$
J(u)=\frac{1}{2} \int_{0}^{1} u^{2}(t) d t
$$

with the constraints

$$
\begin{aligned}
& \dot{x}(t)=A x(t)+b u(t), \\
& F_{j}\left[x\left(t_{j}\right)\right]=\sum_{k=1}^{\gamma_{j}} \alpha_{j k} x_{k}\left(t_{j}\right)-r_{j}=0, \\
& 1 \leq \gamma_{j} \leq \alpha, \quad 1 \leq j \leq N,
\end{aligned}
$$

where $A$ and $x(t)$ are as in $(4)$ and $b=(0, \ldots, 0,1)^{T}$.

We can treat the L-g splines by the same method as in the above section. In this case, the Hamiltonian function is

$$
\begin{aligned}
H & =\frac{1}{2} u^{2}(t)+\lambda(t)(A x(t)+b u(t)) \\
& =\frac{1}{2} u^{2}+\lambda_{n} u-a_{0} \lambda_{n} x_{1}-\sum_{j=2}^{n}\left(a_{j-1} \lambda_{n}-\lambda_{j-1}\right) x_{j} .
\end{aligned}
$$

Comparing with (21), we know $\partial H / \partial x=\partial \widetilde{H} / \partial x$, so the optimization problem (34)-(35) and the optimization problem (12)-(14) have the same adjoint differential equation and transversality conditions. Therefore, (25) and (32) still hold. Let $u^{*}(t), x^{*}(t)$ be the solutions of optimization problem
(34)-(35). By the minimum principle, letting $\partial H / \partial u=0$, we can get

$$
u^{*}(t)=-\lambda_{n}^{*}(t)=L s(t), \quad s(t)=x_{1}^{*}(t),
$$

where $s(t)$ is the corresponding L-g spline. From (20), we have $u^{*}(t)=v(t)=L s(t)$.

From (19), (20), (25), (29), (37), and Theorem 1, we can obtain the results as follows.

Theorem 2. Let $s(t)$ be the L-g spline with the EHB functional interpolation constraints (10). Then $s(t)$ has the following properties:

(i) $L^{*} L s(t)=0$, for $t \neq t_{j}(j=1,2, \ldots, N)$;

(ii) $s^{(i)}\left(t_{j}^{+}\right)-s^{(i)}\left(t_{j}^{-}\right)=0, \quad i=0,1, \ldots, 2 n-\gamma_{j}-1, j=$ $2,3, \ldots, N-1$;

(iii) for $u^{*}(t)=L s(t)$,

$$
\begin{aligned}
& u^{*(i)}(a)=0 \quad\left(i=0,1, \ldots, n-\gamma_{1}-1\right), \\
& u^{*(i)}(b)=0 \quad\left(i=0,1, \ldots, n-\gamma_{N}-1\right), \\
& L_{n-i}^{*}\left[u^{*}\left(t_{j}^{+}\right)-u^{*}\left(t_{j}^{-}\right)\right]=\theta_{j} \alpha_{j i} \\
& i=1,2, \ldots, \gamma_{j}, \quad j=2,3, \ldots, N-1 .
\end{aligned}
$$

By Theorem 2, not only are the well-known continuity properties of differential operator interpolating splines given but also a new continuity property (38) is discovered.

Theorem 3. Let $s(t)$ and $\sigma(t)$ be the $L-g$ spline and the LM$g$ spline, respectively, both of them satisfying the interpolating constraints (14) or (35). Moreover, let $u^{*}(t)=L s(t), M \tilde{u}^{*}(t)=$ $L \sigma(t)$. Then there exists $\varphi(t) \in \operatorname{ker} L$, such that

$$
\widetilde{u}^{*}(t)=M^{*} u^{*}(t), \quad \sigma(t)=M M^{*} s(t)+\varphi(t) .
$$

Proof. (27)-(29) and (37) imply $\tilde{u}^{*}(t)=M^{*} u^{*}(t)$. Thus, recalling $L \sigma(t)=M \tilde{u}^{*}(t)$, we can get $L \sigma(t)=M M^{*} u^{*}(t)=$ $M M^{*} L s(t)$. Since $L$ and $M$ are all constant coefficient linear differential operators, they are exchangeable; then $L \sigma(t)=$ $L M M^{*} s(t)$ and hence $\sigma(t)-M M^{*} s(t) \in \operatorname{ker} L$.

Now we complete the proof of Theorem 3.

From the discussion above, we know if we can find the solution $u^{*}(t)$ of the optimal control problem of system (34)-(35) which is determined by one linear differential operator $L$ and subjected to the same constraint conditions as system (12)-(14), the solution $\widetilde{u}^{*}(t)$ of the optimal control problem of system (12)-(14) which is determined by two linear differential operators $L, M$ can be obtained; that is, $\tilde{u}^{*}(t)=M^{*} u^{*}(t)$.

In order to obtain the LM-g spline $\sigma(t)$ by the corresponding L-g spline from (39), it is necessary to consider the explicit expression of $M M^{*}$. From (8) and (33), we can obtain $M M^{*}$ as follows. 


$$
\begin{aligned}
& \text { When } m=2 q \\
& \begin{aligned}
M M^{*}= & \sum_{k=0}^{q}\left[\sum_{i=0}^{k-1} 2(-1)^{i} c_{i} c_{2 k-i}+(-1)^{k} c_{k}^{2}\right] D^{2 k} \\
& +\sum_{k=q+1}^{m}\left[\sum_{i=0}^{m-k-1} 2(-1)^{i} c_{2 k-m+i} c_{m-i}+(-1)^{k} c_{k}^{2}\right] D^{2 k} .
\end{aligned}
\end{aligned}
$$

When $m=2 q-1$,

$$
\begin{aligned}
M M^{*}= & \sum_{k=0}^{q-1}\left[\sum_{i=0}^{k-1} 2(-1)^{i} c_{i} c_{2 k-i}+(-1)^{k} c_{k}^{2}\right] D^{2 k} \\
& +\sum_{k=q}^{m}\left[\sum_{i=0}^{m-k-1} 2(-1)^{i} c_{2 k-m+i} c_{m-i}+(-1)^{k} c_{k}^{2}\right] D^{2 k}
\end{aligned}
$$

\section{Conclusion and Prospect}

According to [5-7], we define the LM-g spline as an integraldifferential operator spline; this integral-differential operator is determined by the inversion of system. The correspondence between LM-g spline interpolating and state-restricted optimal control problems has been used to deduce the structural and continuity properties of LM-g splines. This correspondence is also used to establish the meaningful relationship between LM-g splines and L-g splines.

For our results in this paper, we believe that there are some topics worthy of further consideration. First, under some appropriate conditions, the results can be generalized to variable-coefficient operators $L$ and $M$. Second, the structural and continuity properties of LM-g splines with state constraints can be deduced by optimal control. Finally, the applications of our results in optimal control and digital signal processing need further investigation.

\section{Conflict of Interests}

The authors declare that there is no conflict of interests regarding the publication of this paper.

\section{Acknowledgment}

This research was supported by Research Project of National University of Defense Technology (JC12-02-01).

\section{References}

[1] Y. Wang, Smoothing Splines: Methods and Applications, CRC Press, NewYork, NY, USA, 2011.

[2] M. Egerstedt and C. Martin, Control Theoretic Splines: Optimal Control, Statistics, and Path Planning, Princeton University Press, Princeton, NJ, USA, 2009.

[3] X. J. Zhang and S. R. Lu, "A spline method for computing a class of minimum-energy control for multivariable linear systems," Control Theory \& Applications, vol. 19, no. 1, pp. 61-64, 2002.
[4] G. S. Sidhu and H. L. Weinert, "Vector-valued Lg-splines. I. Interpolating splines," Journal of Mathematical Analysis and Applications, vol. 70, no. 2, pp. 505-529, 1979.

[5] G. S. Sidhu and H. L. Weinert, "Vector-valued Lg-splines. II. Smoothing splines," Journal of Mathematical Analysis and Applications, vol. 101, no. 2, pp. 380-396, 1984.

[6] R. J. P. de Figueiredo, "LM-g splines," Journal of Approximation Theory, vol. 19, no. 4, pp. 332-360, 1977.

[7] H. L. Weinert, U. B. Desai, and G. S. Sidhu, "ARMA splines, system inverses, and least-squares estimates," SIAM Journal on Control and Optimization, vol. 17, no. 4, pp. 525-536, 1979.

[8] R. Kohn and C. F. Ansley, "A new algorithm for spline smoothing based on smoothing a stochastic process," SIAM Journal on Scientific and Statistical Computing, vol. 8, no. 1, pp. 33-48, 1987.

[9] X. Zhang and K. Fang, "On a class of minimum energy controls and generalized splines," Journal of National University of Defense Technology, vol. 15, no. 4, pp. 84-90, 1993.

[10] G. Opfer and H. J. Oberle, "The derivation of cubic splines with obstacles by methods of optimization and optimal control," Numerische Mathematik, vol. 52, no. 1, pp. 17-31, 1988.

[11] S. Fredenhagen, H. J. Oberle, and G. Opfer, "On the construction of optimal monotone cubic spline interpolations," Journal of Approximation Theory, vol. 96, no. 2, pp. 182-201, 1999.

[12] X. J. Zhang, "Generalized interpolating splines with obstacles and optimal control problems with state constraints," Acta Mathematicae Applicatae Sinica, vol. 23, no. 3, pp. 342-350, 2000.

[13] S. Takahashi and C. F. Martin, "Optimal control theoretic splines and its application to mobile robot," Control Applications, vol. 2, pp. 1729-1732, 2004.

[14] M. Alhanaty and M. Bercovier, "Curve and surface fitting and design by optimal control methods," Computer-Aided Design, vol. 33, no. 2, pp. 167-182, 2001.

[15] X. J. Zhang, "Structure and continuity characteristics of operator spline interpolations associated with invertible linear systems," Mathematica Numerica Sinica, vol. 23, no. 2, pp. 145-154, 2001.

[16] X. Zhang and X. Liu, "Derivation of structural characteristics of differential operator interpolating splines by the criteria of optimal control," Control Theory \& Applications, vol. 28, no. 6, pp. 851-854, 2011.

[17] X. Zhang, "On the inversion of linear systems," Journal of National University of Defense Technology, vol. 20, no. 2, pp. 109113,1998 . 


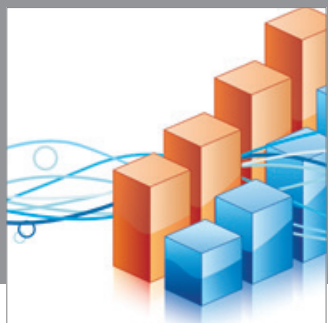

Advances in

Operations Research

mansans

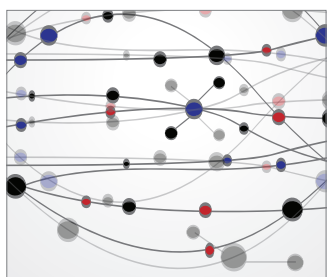

The Scientific World Journal
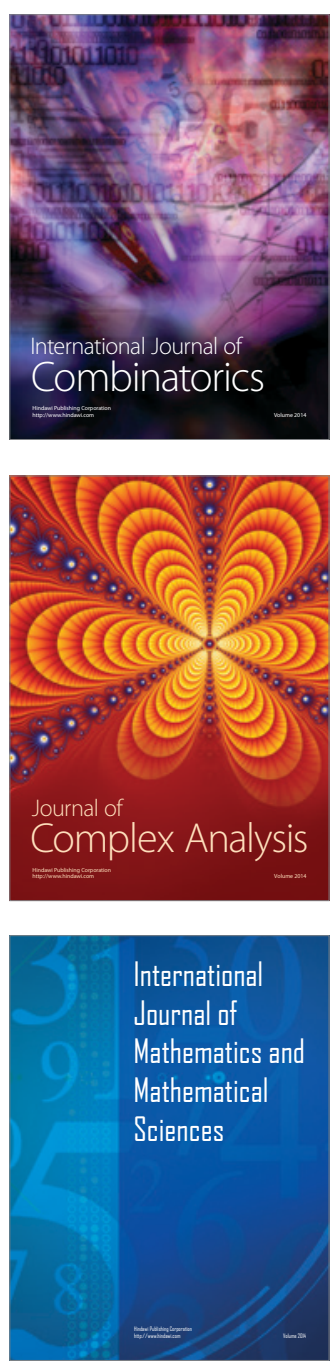
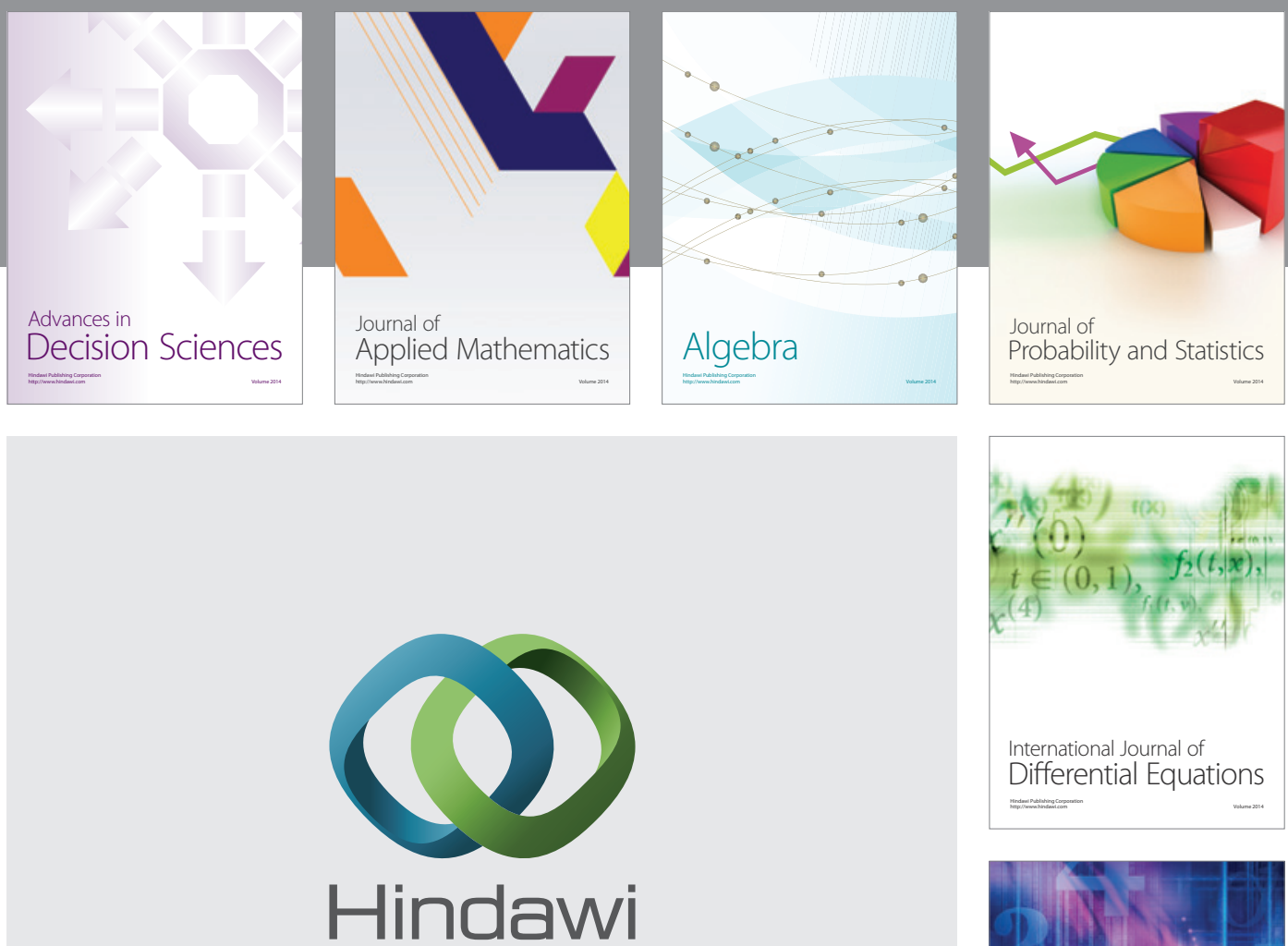

Submit your manuscripts at http://www.hindawi.com
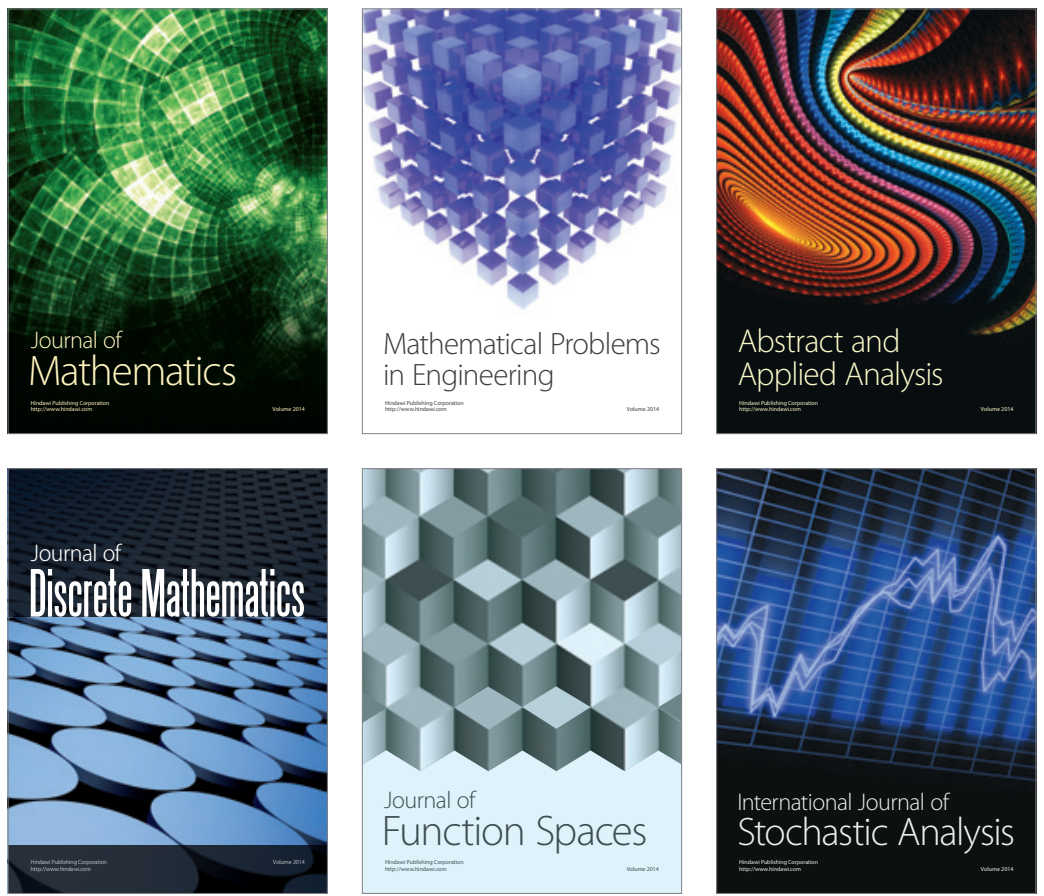

Journal of

Function Spaces

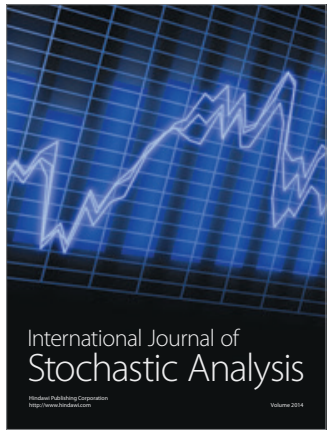

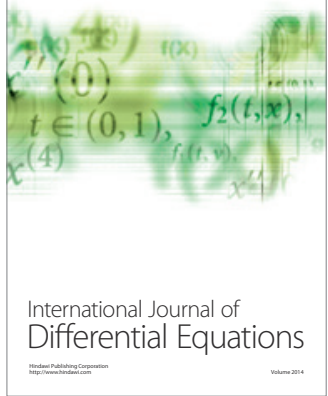
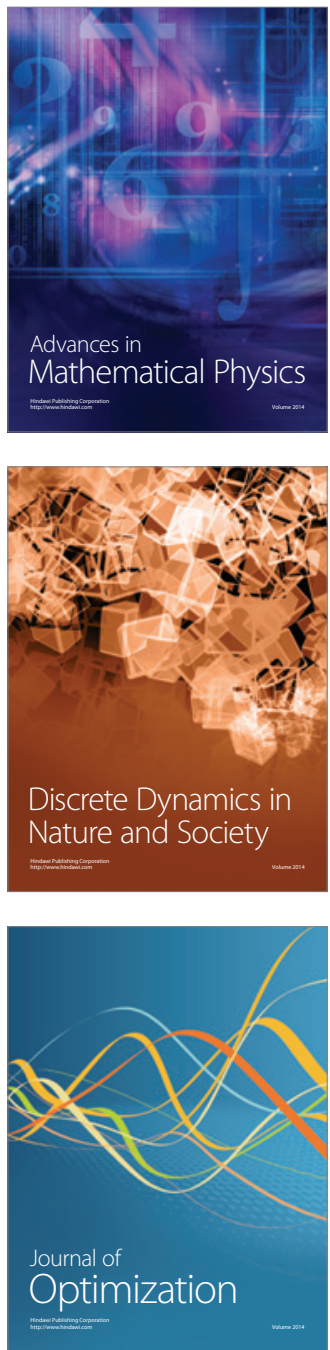